\title{
Baclofeno intratecal para tratamiento de dolor y espasticidad en síndrome de persona rígida: reporte de un caso
}

\author{
Intratecal baclophene for the treatment of pain and \\ spasticity in stiff man syndrome: a case report
}

\author{
J. E. Pérez Meléndez y J. R. Hernández Santos²
}

${ }^{1}$ Residente del Curso de Alta Especialidad de Algología Intervencionista. ${ }^{2}$ Jefe del Servicio de Clínica de Dolor. Centro médico Nacional "20 de Noviembre" del Instituto de Seguridad y Servicios Sociales de los Trabajadores del Estado. Ciudad de México. México

\section{ABSTRACT}

Objective: To present the case of a 32-year-old female patient with a diagnosis of stiff person syndrome, with multiple treatments for pain and spasticity without response, with an intrathecal baclofen test with a positive response, so a definitive implantable device was placed for administration of intrathecal medication (baclofen), performed at the National Medical Center November 20 of the ISSSTE with an improvement of more than $80 \%$ in terms of pain, decreased spasticity.

Methodology: Presentation of clinical case, descriptive and observational study carried out in the National Medical Center November 20, of Mexico City, with follow-up of the patient and its evolution for a period of 12 months between 2017 and 2018.

Results: After the trial with a single dose of intrathecal baclofen, the permanent device was placed for administration of intrathecal baclofen, in the last consultation with a dose of 300 micrograms a day, with an improvement of pain of more than $80 \%$ and with improvement of $100 \%$ in spasticity and overall improvement of the symptoms and with the reincorporation to his habitual life.

Conclusions: The use of intrathecal baclofen should be considered an optimal and real alternative for the treatment of rigid person syndrome through intrathecal administration in Mexico.

Key words: Stiff person syndrome, baclofen, intratecal, pain, spasticity.

Pérez Meléndez JE, Hernández Santos JR. Baclofeno intratecal para tratamiento de dolor y espasticidad en síndrome de persona rígida: reporte de un caso. Propuesta de algoritmo. Rev Soc Esp Dolor 2019:26(6):368-371

\section{RESUMEN}

Objetivo: Presentar el caso de una paciente femenina de 32 años con diagnóstico de síndrome de persona rígida, con múltiples tratamientos para el dolor y espasticidad sin respuesta a los mismos, con una prueba por vía intratecal de baclofeno con respuesta positiva, por lo que se colocó un dispositivo implantable definitivo para administración de medicamento intratecal (baclofeno), realizada en el Centro Médico Nacional 20 de noviembre del ISSSTE con una mejora de más de $80 \%$ en cuanto al dolor, disminución de la espasticidad.

Metodología: Presentación de caso clínico, estudio descriptivo y observacional realizado en el Centro Médico Nacional 20 de Noviembre, de la Ciudad de México, con seguimiento a la paciente y su evolución por un periodo de 12 meses entre los años 2017 y 2018.

Resultados: Posterior a la prueba con dosis única de baclofeno intratecal se realiza colocación del dispositivo permanente para administración de baclofeno intratecal, en la última consulta con dosis de 300 microgramos al día, con una mejora del dolor de más del $80 \%$ y con mejora de $100 \%$ en la espasticidad y mejora en general de la sintomatología y con la reincorporación a su vida habitual.

Conclusiones: El uso de baclofeno intratecal se debe considerar una alternativa óptima y real para el tratamiento del síndrome de persona rígida mediante la administración por vía intratecal en México.

Palabras clave: Síndrome de persona rígida, baclofeno, intratecal, dolor, espasticidad.

Recibido: 23-05-2018

Aceptado: 09-09-2019

Correspondencia: José Edgar Pérez Meléndez epm44@hotmail.com 


\section{PRESENTACIÓN DEL CASO CLÍNICO}

Paciente femenina de 32 años, sin antecedentes de patología crónico-degenerativa, diagnóstico de SPR con pruebas anticuerpos antidecarboxilasa glutámica ácida (antiGAD) positivas. Este padecimiento es una patología neurológica poco frecuente, descrita en la clínica Mayo por Moersch y Woltman en 1956 (1). La prevalencia estimada del SPR es entre 1-2 casos por millón de habitantes, aunque esta cifra podría estar subestimando casos sin diagnóstico, por lo que no se toman en consideración como parte de las estadísticas, debido a lo raro de la patología y el desconocimiento sobre el diagnóstico de la misma (2-4). La enfermedad se describe como de etiología desconocida, caracterizada por la rigidez dolorosa, fluctuante y progresiva de los músculos axiales y de las extremidades, con ausencia de signos de enfermedad cerebral o de la médula espinal, pero con la actividad electromiográfica continua, así como con fibrilaciones y fasciculaciones $[2,5-7)$ que conducen progresivamente a la invalidez, comorbilidad asociada y muerte por complicaciones. Los síntomas generalmente comienzan entre la cuarta y quinta décadas. Afecta a mujeres más que a hombres, relación 2:1 $[8,9)$ (Figura 1).

En la actualidad se considera totalmente una patología autoinmune en donde están presentes los anticuerpos antidecarboxilasa glutámica ácida (antiGAD) positivos en solamente un $60 \%$ de los casos; estos anticuerpos influyen en la transmisión gabérgica. Una parte de los afectados tiene anticuerpos antianfifisina asociados a la presentación de cáncer de mama y pulmón; también se relaciona en portadores de diabetes tipo 1, miastenia gravis, timoma, epilepsia, vitíligo, anemia perniciosa y fracaso suprarrenal, entre otros (10-12).

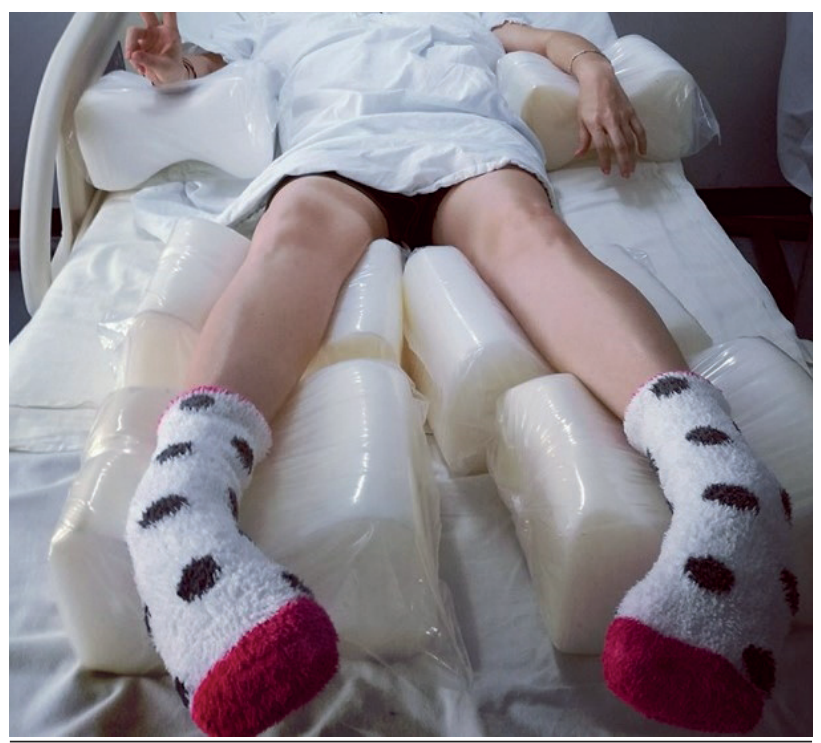

Fig. 1. Paciente con secuelas de la enfermedad, rigidez en miembros pélvicos y postrada en cama con imposibilidad a la deambulación.
Se estiman reportes de 300 casos en el mundo con 3 diferentes presentaciones clínicas de acuerdo con el predominio de los síntomas: la forma típica o síndrome con el tronco rígido (en inglés, Stiff trunc syndrome), el síndrome de las extremidades rígidas [Stiff limbs o legs syndrome], y la encefalomielopatía con rigidez y mioclonía $(13,14)$. Los criterios clínicos para el diagnóstico de la forma típica de la enfermedad son: clínica de comienzo lento y progresivo, rigidez muscular progresiva de predominio axial y espasmos musculares dolorosos. Existen variantes del síndrome de la persona rígida típico, entre las que podemos encontrar: el jerking stiff-man, el focal stiff-man y el paraneoplastic stiff-man $(12,14)$.

El diagnóstico continúa en la actualidad siendo un reto para los médicos involucrados en el caso; se determina mediante la presencia de criterios clínicos y de anticuerpos antiglutamato decarboxilasa y el electromiograma con alguna anormalidad, mientras que la resonancia magnética nuclear craneal y medular, así como el análisis del líquido cefalorraquídeo, resultan en la mayoría de los casos normales $(11,15)$.

El pronóstico de esta patología es incierto y variable; en la actualidad no hay ningún indicador confiable de la velocidad y la gravedad de la aparición de la enfermedad; se han reportado complicaciones comunes como ansiedad, depresión, fracturas patológicas, rupturas musculares y disfagia y, en etapas avanzadas, puede llegar a haber una afectación del sistema respiratorio. La paciente estuvo hospitalizada 10 meses previos a la colocación del dispositivo permanente, fue tratada con dosis de analgésicos opioides vía intravenosa para el dolor así como múltiples tratamientos (inmunoglobulinas, esteroides, baclofeno vía oral, el cual provocó retención urinaria y cuadros de hipotensión severa] para el dolor y espasticidad sin respuesta a los mismos, se realizó una prueba por vía intratecal de baclofeno con dosis de 50 microgramos con respuesta positiva, considerada esta disminución de la espasticidad en un periodo de 24 horas y con disminución de dolor en un $50 \%$ aproximadamente, por lo que se decidió colocar en octubre de 2017 el dispositivo de infusión intratecal de fármacos modelo Synchromed II, de $20 \mathrm{ml}$ de reservorio, el cual se realizó por abordaje percutáneo, en sala de fluroscopia con sedación consciente de la paciente; se colocó a la paciente en posición de decúbito prono con sistema Spine para rectificación de la columna lumbar; se realizó monitoreo tipo 1, posteriormente mediante la técnica habitual se introdujo catéter hasta llegar la punta a plataforma inferior de T8 y se tunelizó el catéter hasta región abdominal en cuadrante superior derecho en donde se realizó la colocación del reservorio definitivo a nivel subcutáneo finalizando el procedimiento sin complicaciones ni incidentes (Figura 2), con dosis iniciales de 25 microgramos cada 24 horas, durante 4 días, con una mejora en la espasticidad de un $50 \%$ y de dolor en un $70 \%$. En esta fecha se realizó aumento de la dosis de manera progresiva hasta llegar a 150 microgramos con los que se egresó en noviembre el 2017, ya habiendo iniciado rehabilitación física, con disminución de la espasticidad en más de un $80 \%$ y de dolor en más de un $70 \%$, sin otros medicamentos agregados a su egreso. En la actualidad con un segui- 


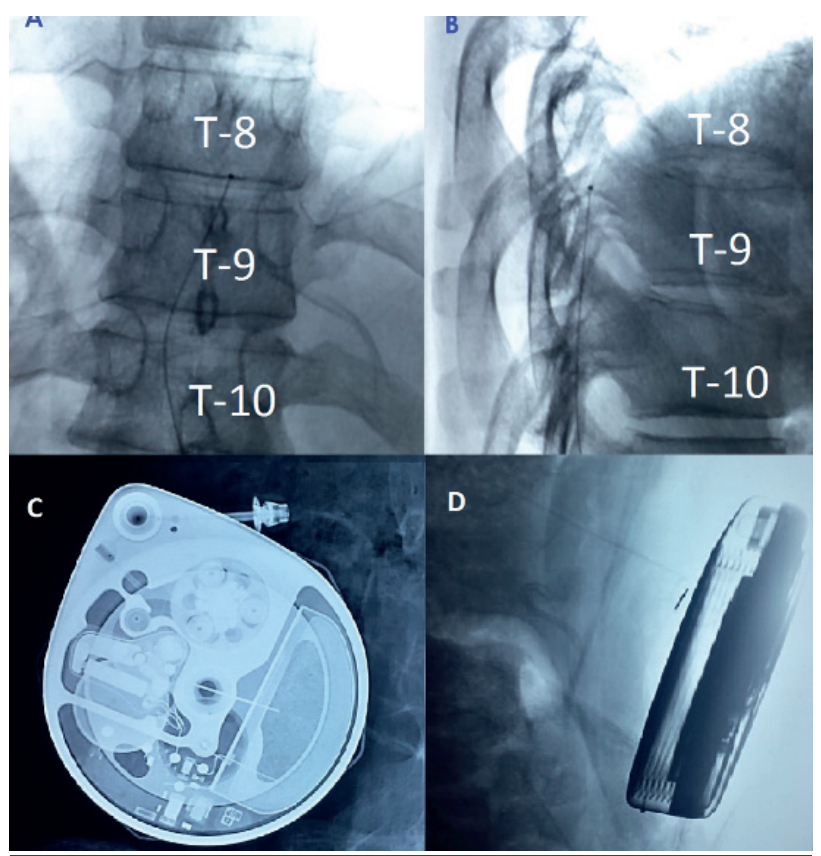

Fig. 2. Imágenes de la colocación del dispositivo de infusión intratecal de fármacos modelo Synchromed II, de 20 $\mathrm{ml}$ de reservorio. A y B: imagen del catéter; C y D: imagen del dispositivo.

miento por un periodo de 12 meses se encuentra con dosis de 300 microgramos cada 24 horas.

\section{RESULTADOS}

Posterior a seguimiento por 18 meses durante los cuales se incrementó la dosis de manera progresiva hasta llegar en la última consulta en febrero del 2019, con dosis de 300 mcgs cada 24 horas en infusión, sin presencia de efectos adversos al medicamento, con EVA 1/10, EVERA leve, con deambulación por cuenta propia, mejora de un $90 \%$ en relación con el dolor y mejora de un $100 \%$ en la espasticidad. Última crisis de espasticidad presentada hace más de 6 meses, sin necesidad de aumento de la dosis (Figura 3).

\section{DISCUSIÓN}

El presente trabajo tiene la intención de presentar una entidad clínica rara e inusual como lo es el SPR además de confirmar el uso de un medicamento el cual no se cuenta por vía intratecal en México y en muchos otros lugares del mundo, pero coincide con los estudios de Dalakas (5) y Jairala [6] en cuanto a la presentación clínica y al manejo médico establecido.Sin embargo, no presentó la mejoría esperada con el uso de los tratamientos convencionales, por lo que se determinó el uso del dispositivo vía intratecal de manera definitiva como una última opción, coincidiendo con el trabajo de Kamensek (16), en donde se concluye que a pesar de no haber ensayos a largo plazo, los resultados de dicho estudio

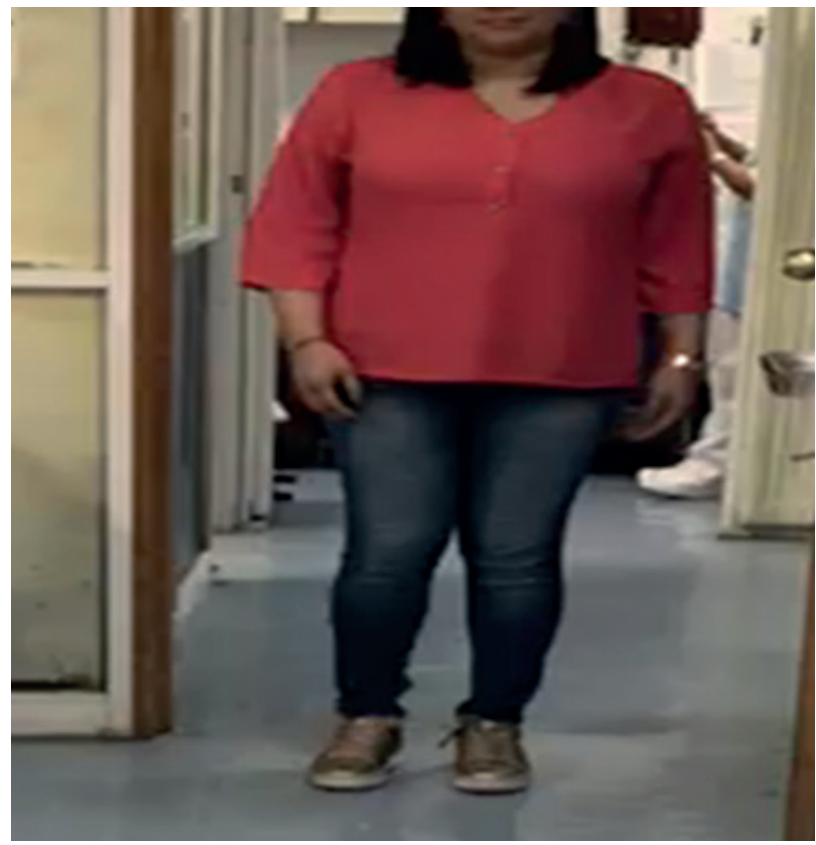

Fig. 3. Paciente en seguimiento con deambulación sin necesidad de ayuda, sin presentar dolor y sin crisis de espasticidad.

sugieren que el baclofeno intratecal es una terapia eficaz para la espasticidad médicamente intratable debido a SPR, y la reducción de síntomas se puede lograr sin comprometer la ambulación y coincide con nuestro caso en donde concluimos que el baclofeno intratecal se presenta como una opción terapéutica eficaz para la espasticidad refractaria o espinal, alcanzando niveles terapéuticos en el líquido cefalorraquídeo con dosis mucho más pequeñas en comparación con las medicaciones orales mientras reduce los efectos secundarios sistémicos $(10,15)$. Las posibles complicaciones de la terapia ITB están bien descritos, y la manera de disminuir al mínimo estas complicaciones consiste desde la adecuada selección del paciente hasta el cuidado de todo el procedimiento, el cual deberá ser realizado por personal con experiencia en este tipo de dispositivos $(15,16)$. En nuestro caso, y con el seguimiento posterior de más de 6 meses con una mejora de un $100 \%$ en la espasticidad y la ausencia de crisis, nos dan la indicación sustentada en la bibliografía y en la escasa experiencia de nuestro país para el manejo definitivo de esta patología mediante el uso de baclofeno intratecal.

\section{BIBLIOGRAFÍA}

1. Cabo López, I, Negueruela López M, García Bermejo P, Zamarbide Capdepón I, García Ruiz PJ, Durán Martínez $P$, et al. Síndrome de la persona rígida: A propósito de un caso. Rev Neurol. 2008;47(5):249-52. D0I: 10.33588/ rn. 4705.2008154.

2. Dalakas MC. Stiff-person Syndrome: Advances in Pathogenesis and Therapeutic Interventions. Curr Treat Options Neurol 2009; 11(2):102-10. 
3. Pagano MB, Murinson BB, Tobian AA, King KE. Efficacy of Therapeutic Plasma Exchange for Treatment of Stiffperson Syndrome. Transfusion. 2014;54(7):1851-6. DOI: $10.1111 /$ trf. 12573.

4. Moersch FP, Woltman HW. Progressive Fluctuating Muscular Rigidity and Spasm ("Stiff-man" Syndrome); Report of a Case and Some Observations in 13 Other Cases. Mayo Clin. 1956;31(15):421-7.

5. Dalakas MC, Fujii M, Li M, Lutfi B, Kyhos J, Mcelroy B. Highdose Intravenous Immune Globulin for Stiff-person Syndrome. N Engl J Med. 2001;345(26):1870-6. DOI: 10.1056/NEJMoaO1167.

6. Jairala-Quade L, Sánchez-Espinoza J, León E, Naranjo J, Vásquez E. Síndrome de la persona rígida. Rev Neurol. 2009;48(4): 16-28.

7. Hijazi J, Bedat-Millet AL, Hannequin D. Stiff-person Syndrome and other Neurological Disorders Associated with anti-GAD Antibodies. Rev Med Interne. 2010;31(1):23-8. DOI: 10.1016/j. revmed.2009.01.012.

8. Hadavil S, Noyce AJ, Leslie RD, Giovannoni G. Stiff-man Syndrome. Pract Neurol. 2011;11(5):272-82. DOI: 10.1136/ practneurol-2011-000071.

9. Rakocevic G, Floeter MK. Autoimmune Stiff-person Syndrome and Related Myelopathies: Understanding of Electro- physiological and Immunological Processes. Muscle Nerve. 2012;45(5):623-34. DOl: 10.1002/mus.23234.

10. Ciccoto G, Blaya M, Kelley RE. Stiff-person Syndrome. Neurol Clin. 2013;31(1):319-28. DOl: 10.1016/j.ncl.2012.09.005.

11. Murinson BB, Wilson RK. Stiff-person Syndrome. Neurologist. 2004;10(3):131-7.

12. Teresa N, Neame R. Síndrome de Stiff Person, Departamento de Farmacología Molecular y Fisiología, Universidad del Sur de la Florida [Internet]. [Citado 28 Sep 2009]. Disponible en: http:// emedicine.medscape.com/ article/1172135-diagnosis.

13. Hagiwara H, Nakatani SE, Sakai K, Ugawa Y, Kusunoki S, Kanazawa I, et al. Stiff-person Syndrome Associated with Invasive Thymoma: a Case Report. J Neurol Scien. 2001;193(1):59-62.

14. McKeon A, Robinson MT, McEvoy KM, Matsumoto JY, Lennon VA, Ahlskog JE, et al. Stiff-man Syndrome and Variants: Clinical Course, Treatments, and Outcomes. Arch Neurol 2012;69(2):230-8. DOI: 10.1001/ archneurol.2011.991.

15. Abbatemarco JR, Willis MA, Wilson RG, Nagel SJ, Machado AG, Bethoux FA. Case Series: Intrathecal Baclofen Therapy in Stiff-Person Syndrome. Neuromodulation. 2018;21(7):6559. DOl: 10.1111/ner.12765.

16. Kamensek J. Continuous Intrathecal Baclofen Infusions. An Introduction and Overview. Axone 1999;20(4):93-8. 